International Journal of Engineering \& Technology, $7(2.7)(2018) 696-704$
International Journal of Engineering \& Technology
SPC
Website: www.sciencepubco.com/index.php/IJET
Research Paper

\title{
Innovative Data Transmission and Routing Strategies of Wireless Body Area Sensor Networks: Taxonomy, Review, Issues and Constraints of State of the Art Contributions in Recent Literature
}

\author{
R.Anirudh Reddy ${ }^{1 *}$, Dr.N.Venkat $\operatorname{Ram}^{2}$ \\ Research Scholar ${ }^{1}$, Department of ECE, Professor ${ }^{2}$, Department of ECSE \\ Koneru Lakshmaiah Educational Foundation, Vaddeswaram, Guntur, Andhra Pradesh, India-522502 \\ *Email: rikkulaanirudh@gmail.com
}

\begin{abstract}
Wireless body area sensor networks (WBASN) is an effective solution that has proposed in terms of improving the solutions and their varied benefits that have been achieved from the usage of WBASN solutions in communication, healthcare domain. From the review of stats on rising number of wireless devices and solutions that are coming up which is embraced by the people as wearable devices, implants for medical diagnostic solutions, etc. reflect the growing demand for effective models. However, the challenge is about the effective performance of such solutions with optimal efficiency. Due to certain intrinsic factors like numerous standards that are available, and due to the necessity for identifying the best solutions that are based on application requirements. Some of the key issues that have to be considered in the process of WBASN are about the impacts that are taking place from the wireless medium, the lifetime of batteries in the WBASN devices and the other significant condition like the coexistence of the systems among varied other wireless networks that are constituted in the proximity. In this study, scores of models that have propose pertaining to MAC protocols for WBASN solutions have reviewed to understand the efficacy of the existing systems, and a scope for process improvement has explored for conducting in detail research and developing a solution.
\end{abstract}

Keywords: IEEE 802.15.6, Medium access control, Physical Layer, Routing, Wireless Body Area Networks, Wireless Sensor Networks, Energy-Efficiency, MAC Layer, Physical Layer, Sensors, Routing Protocols, Power saving techniques, Health care system.

\section{Introduction}

The Emergence of WBASN (Wireless Body Area Sensor Networks) has transformed the way communication among the application systems and its integral devices take place. There are revolutionary developments that have taken place in the WBASN, is profoundly adapted in healthcare Smart cities, and fitness [1], and in varied other range of IoT applications.

WBASNs play a vital role in health care and in other such industrial applications [2]. The key advantage of adapting the WSBNs in health care monitoring is that it did not interrupt the normal functions of the human system or be any inconvenience to the customers and also it ensures that adequate inputs required from the human body are relayed to the application system more effectively. Using BSN it is very much feasible to address monitoring of heart rates, functions of the body, brain activities, body temperature, muscle motions etc in the more accurate manner [3]. It is very essential that the solutions adapted have to be more accurate in addition to adherence for health checking and intimation conditions.

It is very much feasible to evaluate the person's health conditions like B.P (Blood Pressure) using WBASN based on modified volume oscillometric technique that mitigates the need to deploy any kind of inflatable pressure cuff, mobile device [4] or any kind of hearing aids. In the other dimension, there is growing demand for usage of wearable devices in the sports and training for evaluating distinct metrics. For instance, usage of WBASN for evaluating the sweat rate or heartbeat rates during varied conditions of training is gaining momentum. [5] Using of WBSN has provided scope for targeting on accurate information essential for managing the treatment even from home for certain critical diseases like heart attacks, Parkinson, sleep apnea etc [3].

During the post-recovery during the rehabilitation process, there is need for continuous monitoring. All type of body signals and health conditions of the patient are to collect monitored. WBASN provides valuable information like audio feedback, real-time images, and important information that are essential to be gathered. WBASN can be collecting the data from the individual patients and can monitor the remote patients too.

Such obstacles are widespread adoption of BASN. Authors of a study [6] were of viewpoint that both the manufacturers and the users require orientation adoption of the widespread BASN. Critical requirements comprise safety for humans from the implanted and wearable sensor nodes. The authors of [6] as depicted in Figure 1 discuss WBASN environment, it has highlighted significant challenges encountered by WBSAN. 


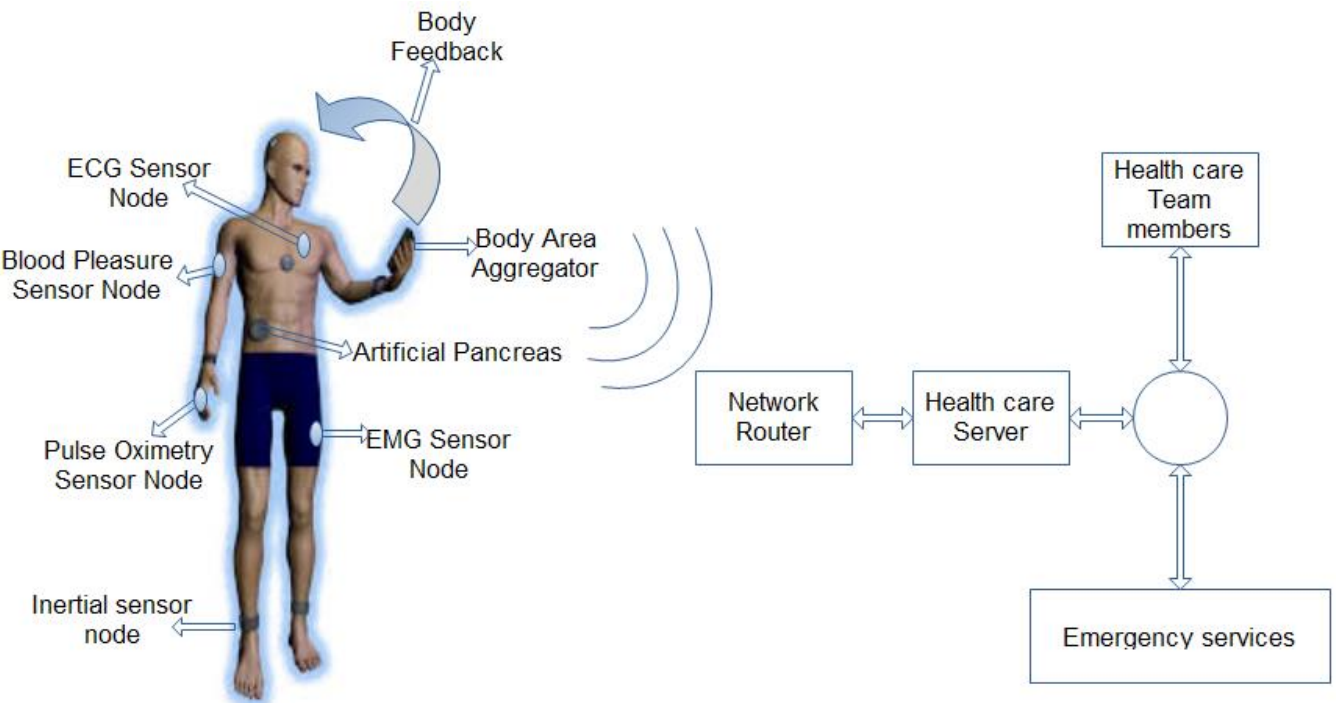

Figure 1: Indicates of the feasible positioning of sensors nodes

WBASN physical hardware nodes whilst emerging in too much smaller devices, the requirements in terms of power efficiency to handle the conditions are on rising [7]. To address such power constraints, a number of power saving and security techniques were introduced for addressing conflicting goals. Categorically, in the case of rehabilitation applications like WBASN are adapted for time-dependent data processing and acknowledgment provisioning. In addition, the health application imposes mandate requirements for assuring QoS.

Firmware applications focus on data collection at sensor node level, data processing, and power saving techniques was adapted. Many of the hardware and software solutions have targeted on energy levels of sensor node and consuming techniques like the instant power and the frequency scaling, power-aware scheduling etc. Many selective approaches were dependent on the hardware choices like the low-power radios and the custom ultralow power subsystems or components [7].

The Figure 2 details the power consumption level of radio transceiver established in the wireless sensor nodes and if such power consumption can reduce, it can be more resourceful outcome.

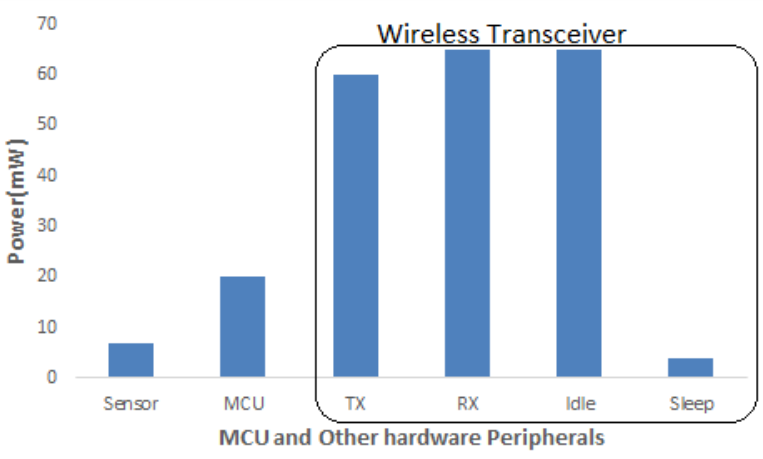

Figure 2: Indicative inputs on power consumption in wearable nodes [7].

Based on varied activities and application goals, devices switch amidst the varied power saving policies pertaining to distinct setup of hardware components (like transceiver power states, microcontroller hardware configuration, or the sensor-sampling rate).

\section{Nomenclature of the Wireless Body Area Sensor Networks}

Patient health monitoring nodes usually exchange the healthrelated information like Bluetooth, ZigBee, surveillance solutions, and WLAN networks. Significant developments are taking place in the consumer electronic solutions that are enabling better quality of life [8].

In the WBASN solutions, there are profound developments that anticipated in usage of the solutions for healthcare domain. With the rising trends of internet revolutions, there is exponential growth in the demand for such solutions [9]. WBASN are very effective in handling the information and communication solutions Certain functions like sampling, real-time data relay, information processing, facilitating communications performed without any interruptions [9]. In tracking the physiological parameters and in terms of offering flexibility and mobility to the uses, adapting WBASN can offer significant results. Features of offering data from large time intervals, and in improved frequencies can enable the doctors to have more insightful information pertaining to patient's status [10]. Challenge is about engaging in practical implementation and adaptability of such solutions.

Such conditions lead to design and implementation related challenges, as the objective for such system has minimum delay, network lifetime and maximum throughput and are in terms of reducing unwanted communication pertaining to energy consumption. In addition, the user-oriented requirements for adoption of WBSAN are about privacy, ease of implanting, value of systems, safety conditions [9].

\subsection{Topology used in WBASN}

IEEE 802.15.6 has adapted WBSN for both the one-hop and twohop star-based topology to ensure effective communication based on positioning at strategic location [11]. Communication methods that used over the beacon or non-beacon models, works based on the beginning and ending at a super frame for enabling network association and kind of device synchronization.

CSMA/CA used for non-beacon mode depending on essentiality [12]. In the case of WBSN, the coordinator is termed as sink node and the ones that one-hop start topology and multi-hop architecture nodes profoundly connected and communicated to varied level of communication access points, depending on the other sensor nodes.

For handling compliant communication, two hops are adapted as per the IEEE standards, and some of the proprietary systems that could adapt more than two hops considered in the process. Despite all such developments, the issue of interoperability is a major concern because of challenges resulting due to standard compliant. 


\subsection{WBASN Communication Architecture}

Mainly WBASN communication architecture can categorize into three groups,

Tier- 1 is profoundly over the network interaction of nodes and the transmissions ranges that are impacting the human body.

Tier-2 is an effective medium amidst Ps and the other access points (Aps). The Aps are integral to the infrastructure that shall position for a dynamic environment. Tier-2 communication shall enable the WBSN's correspondence to varied networks that can be easily accessed [8]. Some of the key dimensions that considered as subcategories towards inter- WBASN communication are the ad-hoc oriented architecture and the infrastructure-based architecture [8].

In Tier-2, the emphasis is about usage of metropolitan areas and the gateway kind of PDA, which can be resourceful for bridging gap amidst the tier- 2 and tier- 2 solutions. The database is among the most effective components for addressing tier-3, which usually restores necessary information for further course of action.

\subsection{Challenges in QoS WBASN}

Some of the key issues in terms of addressing QoS for $W B A S N S$ are:

\subsubsection{Physical Layer Challenges}

PHY layer of protocols designed for addressing the power consumption while ensuring that the reliability issues of the system not compromised. However, the crux in the case of current solutions are about minimizing the average power consumption by the radio during the phase of active and sleep modes [6]. In addition to that, the interface is the other major setback in the case of WBASN systems, wherein the developments emerging because of improved co-existence. The issue of off-body interference resulting because of collision with the external sensors are also an intrinsic issued encountered in the process [13].

\subsubsection{MAC Layer Challenges}

Mechanisms that are integral to IEEE 802.15.6 not developed relying upon MAC protocols and the emphasis on addressing the interoperability issues. Certain solutions like the message exchange protocols, packet formats evaluated as a scope for research. In the conditions wherein, the reliability not attained from one-hop start topology, relays were adapted for attaining the outcome [8]. WBASNs need categorical range of QoS requirements for adhering to the MAC proposal [8].

\subsubsection{Transport Layer (QoS) Challenges}

QoS requirements of the application in WBASNs were to be addressed ensuring that no plunge in the performance and without any complexities. In addition, in real-time scenario, some of the WBAN impacted in terms of loss and relay. Even the issues pertaining to limited memory impacts to great extent the outcome that expected from the process. At times, the QoS features like the reliability; bandwidth might impact the process for performance related challenges. For attaining the lower level of packet losses, the transmit power has to improve that could result in higher levels of power consumption. The summary of layer level challenges has given briefly in Figure 3.

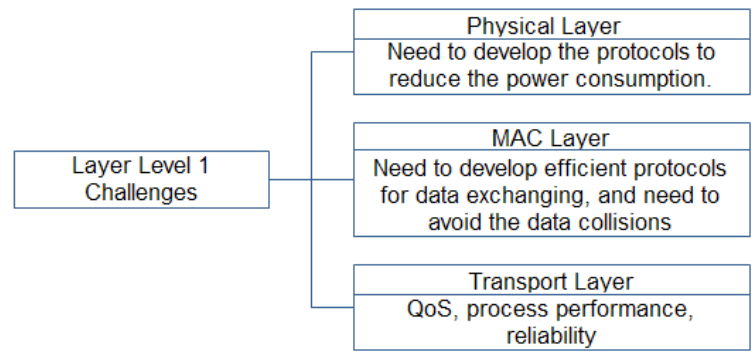

Figure 3: Different types of challenges in layers of Wireless body area sensor network

\subsubsection{Sensor fusion Challenges}

Many of the contemporary researches were focusing upon upcoming research challenges and directions, specifically in terms of Autonomic WBASN s, Cloud-oriented BANs, Collaborative WBASNs, and Context-aware WBASNs, which discussed in below and represented in Figure 4.

Autonomic WBASNs: These types of WBASN systems are predominantly a sensor self-healing, configurable, optimizes solutions. Every property requires due assessment using multi-sensor node fusion methods. Sensor Self-maintenance profoundly based on wireless sensor node calibration and real-time sensor datafiltering [14], wherein the self-optimization shall ensure dynamic model evolution. Even the process of self-configuration shall handle the wearable sensors replacement or displacement.

Context-aware WBASNs: In the instance of a change in the context, there is imperative need for multi-sensor nodes fusion techniques to point out such type of changes, as it impacts the properties of the methods like accuracy issues. The process of transfer learning model, which is integral to the frameworks, using the transfer learning could consider WBASN to adapt varied contexts by extracting and transfer of data from one context to the other. In an illustrative scenario, activity of sensor node classification can process from one context to the other [15]. In the instance of datasets not annotate, methods like sensor profiles are required to encode the background knowledge. The design can facilitate every sensor in the source domain dataset for matching the counterpart over the target domain. Certain facets like position of a patient body sensor node attached might be very essential for a sensor profile.

Improving the Battery life: In designing of sensors, battery is one of the significant concerns. Profoundly, the battery lifetime and discharging time of a sensor node depend on the number of sensors, which connected to hardware. More number of tasks processors can handle the quantum of energy requirements in simultaneous manner. Hence, effective solutions with more efficiency of power management is integral to WBASN domain [16]. WBASN as a solution comprises concern for self-charging or power drawn from the external resources, and even from the WiFi signals.

Collaborative WBASNs: Collaborative WBASN [17] are the BSNs that are able to collaborate with every other for fulfilling common goal. Multi-sensor nodes data fusion is one of the common CBSNs that are integral to joint data analysis like synchronized data integration and classification, filtering, time-dependent solutions. CBSNs can usually program using C-SPINE [18], wherein the extension of SPINE framework is imperative [19]. Using the C-SPINE, the e-shake CBSN systems were developed [20]. E-Shake is a solution developed using multi-sensor data fusion scheme for performing automatic detection of handshakes amidst two individuals and towards capturing the relational responses.

Cloud-based Wireless Body Area Networks: CABANs have overlay infrastructures that integrate BANs atop the cloud systems 
for offering effective and efficient sensor stream for gathering patient health care node stream management, persistent body sensor data collection, storage, and decision making, functionoriented body analysis, enhanced visualization, and multi-layer security. A popular illustration of CABAN is the Body Cloud [21] which supports in generated new community-oriented WBASN systems, which will raise vivid range of interesting issues like definition of novel fusion methods for community oriented WBASN. In addition, the high-volume of streamed data for fusion algorithms offer accurate output and time-based data processing management of multiple sensors.

Sensor Design: WBASN technologies offer significant opportunities in healthcare monitoring. In addition, there are certain challenges that are emerging with the development. One of the significant issues that envisaged is with sensor design, wherein researchers focus on integration of more sensors to one node whilst addressing less power [22]. Multi-parameter measurement is one of the intrinsic factors that is highly cost-efficient; it might be more expensive than single sensor node. It also needs much more accuracy, firmware reliability, and robustness than the nodes, which contains single sensor.

Issues pertaining to handling novel range of handheld devices: Smart phones [23] and other such range of computing devices are turning out to be base stations for WBASN developers. The handheld devices are more adaptable, and the constraint is in terms of limited capacity in terms of handling. In addition, until one is acquainted with the process of handling, managing the handheld devices becomes much challenging.

Choosing the best routing path: In the case of patients staying outside the direct monitoring of hospitals, the key issue is about executing the task of monitoring in a different manner. The key concern is the task shifting for selecting best routing path [24]. For instance, patient's home can be base station forwarding data using the WLAN to the remote servers. However, in such cases, there are potential chances of base stations becoming less active and the transmission of data might take place at lower frequency levels using WWAN and GPRS. Involvements of some nearby relay stations are essential for wireless networks to realize long-distance wireless transmissions.

WBASN has certain constraints in terms of data collection reliability, privacy, and patient identification related issues for addressing wearable sensing system to ensure continuous activity monitoring [25].

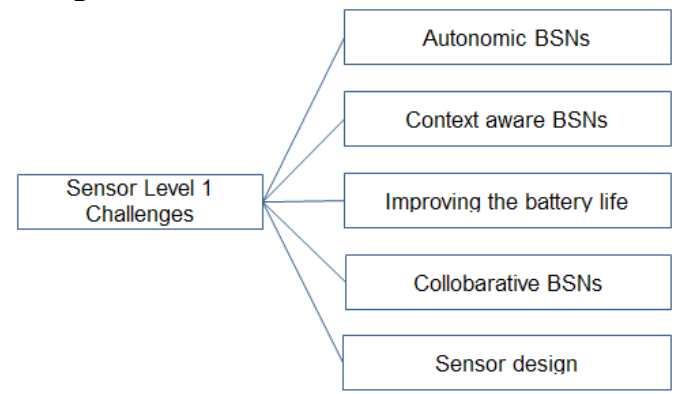

Figure 4: The sensor level challenges of Wireless body area sensor network

\section{Contemporary Affirmation of Benchmark- ing Routing Strategies in WBASN}

In the case scenario of WSNs and MANETs, routing protocol systems were reviewed in an extensive manner and from the studies, it is evident that WBASN has significant impact in terms of constraints of design over the routing protocols, which results in constrains like routing performance issues.

Implementation of WSNs energy, efficient routing protocols are very sensitive in terms of processing the data, memory access, and another such kind of measurements. WSN nodes are homogeneous, the WBASN nodes are heterogeneous, and it has wide range of scope for data rate and energy availability [10], variation in mobility. WBASN routing has to take into account variations in body, limitation of energy resources, and radiation impact over the tissue heating and limited energy resources for adapting available resources to mitigate intervals for charging the batteries, improving the lifespan and towards developing quality system.

Although the properties of WBASN related to MANETs and WSNs, still the unique difference could attribute to contemporary solutions, which are essential in terms of routing protocols.

\subsection{Temperature Based Routing}

Wireless communication solutions vividly used for magnetic and electric fields generation from the radio signals. High level of radiation emission and exposure to such levels of radiation results in rise in the body temperatures of the humans, which might lead to health implications.

Temperature oriented routing algorithms are developed, wherein the emphasis is on reducing hot spots. Levels of heating and radiation absorption over in a human body are certain key aspects to consider in the design of routing protocols. TARA is one of the integral solutions that were proposed, which targets the addressing of temperature issues. However, the reliability and packet loss ratios in addition to low network lifetime are certain integral issues in the model, which addressed in the other model, called LTR (Least Temperature Routing) Algorithm [26]. One of the key challenges is about getting insights in to the temperature phenomenon of each node and the other nodes.

\subsection{Cluster-Based Routing}

Cluster-based routing protocols are adapted in WBASN are adapted in WBASN classify the nodes in to varied clusters. For each of the generated cluster, a cluster-head assigned and used for data transmission from sensor to sink. The key objective of such routing protocols are to mitigate the number of direct data transmissions resulting from sensors to the base station. The other significant challenge is the overhead and delay pertaining to cluster selection, which are major problems for such protocols.

Authors of [27] adapting a data generated protocol using "Anybody" has proposed reduction in the quantum of direct transmissions towards a sensor node base station. However, one of the key constraints envisaged is the energy efficiency issues ignored in the model. The constraints envisaged in the process is addressed in HIT (Hybrid Indirect Transmissions) [28], resulting in improved energy efficiency which is not considered in the process.

In [28] authors focused on the model of data generating protocol that targets in reducing number of direct transmissions towards a sensor node base station and by focusing on multi-hop indirect transmissions towards a cluster and for multiple clusters that are adjacent. Though the high-energy efficiency and network lifetime can envisage, still there are certain factors like network delay envisaged because of HIT and HITm, while addressing dense networks and issues of reliability and conflicting interaction ignored.

\subsection{Probabilistic Routing}

Many alternative routing protocols like the probable of cost factor to account and the work towards handling a route carried out at minimal cost. However, such protocols need varied transmissions for updating link-state information that could turn out to be a constraint in implementing macro range of protocols.

In [29], the authors Introduced ETPA method which means "Energy Efficient Thermal and Power-Aware routing" that offers solution for proposed factors of relative costing solutions. In addition, certain complex factors like depletion time that could result in lasting communication among the nodes considered in WBASNs. 


\subsection{Cross-Layer Routing}

The routing protocols that are of Cross-layer proposed to focus on the issues pertaining to network layers. Though such protocols have very poor energy consumption, still the emphasis could be more about higher path loss and it could even impact over the body motion. Some of the significant solutions like WASP proposed in [30] target to address the issues by focusing on WASP cycles, and towards improving traffic routing.

CICADA which means "Controlling Access with Distributed Slot Assignment protocol" [31] is the other model constituting low energy cross-layer routing protocols specifically proposed for WBASNs, which based on multi-hop TDMS scheduling.

TICOSS (Time zone Coordinated Sleeping Mechanism) [32] targets all nodes as FDD (Full Functional Devices) and it enhances the standards of configuration using the shortest path route to WBASN coordinator. It also preserves energy whilst minimizing hidden terminal data collisions using various $\mathrm{V}$-scheduling that doubles the operational lifetime for IEEE 802.15.4 for busy traffic scenarios and extension of IEEE 802.15.4 for supporting mobility. BIOCOMM [33] is the other cross-layer routing protocol developed for interaction among MAC and network layer over biomedical sensor networks towards optimizing the performance of network. Such interaction attained by using "Cross-layer Messaging Interface" by which the MAC layer relays its status information to the network layer.

\subsection{QoS Based Routing}

QoS oriented routing protocols are among the popular kind of routing protocols. Varied methods were proposed based on the efficiency model and the metrics that could impact the efficient process of routing. A contemporary QoS related routing protocol (LOCALMOR) is proposed in [34] towards improvising the sensor networks utilization of biomedical applications.

The review of contemporary solutions reflects the fact that majority of the studies have focused on routing path and categorically in terms of geographical routing and path routing conditions. Though majority of such constraints were to be addressed using the process of RL-QRP algorithm, impact of such independent distributed reinforcement learning model for QoS route calculations focus on sensor nodes, however, the key constraint is that the proposed solutions lack scope for global optimization towards large-scale network conditions.

\section{Contemporary Literature Review of WBASN}

This section of the paper explores varied types of solutions that try to be energy efficient and over security in the WBSN. In this section, we reviewed on different types of proposed solutions, which are trying to get for energy efficiency, security on WBSN.

\subsection{Energy efficiency mechanisms}

In [35], a system-level mechanism to power consumption proposed. The mechanism combined aspects based on the optimization of power and application. In [36], authors have targeted implementation of energy-efficient transceiver, using injection locked frequency classifier that can conserve energy.

In [37], adaptive frequency-hopping plan is adapted and authors of [38] focused on low power on-body communication was proposed A direct-coupled interface proposed for mitigating ground level electrode considered as integral factor in the study.

In [39], a study of signal-processing mechanisms introduced, where in the framework of QoS regulation-oriented optimization, principles discussed. Authors of [40] have focused on energyefficient cost-effective protocol towards multi-hop WBASN and such protocol adapts the relay position and data routing constraints to improve the longevity of the network.
QoS can consider data-centric routing protocols, and in [41] authors have explored the model of data-centric multi-objective QoS-Aware routing protocol DMQoS. Varied thermal routing protocols used in WBASN. TARA is the first thermal-aware routing solutions in BSNs [26], in which upon receiving a packet for sending, a node selects the next node with minimal temperature. In [26], authors have explored thermal-aware routing protocols LTR and ALTR.

In [28], HIT a cluster-based routing mechanism proposed, in which the routing allowed nodes for parallel and multi-hop sending. WASP and CICADA [42] are two of the cross-layer protocols that designed with MAC and other network layers. Routing of such protocols carried out using spanning tree and time slots. Certain routing protocols are QoS-aware. A data-centric multiobjective QoS-Aware routing protocol discussed in [41]. Authors of [43] discussed model of QoS reinforcement learning using the routing protocol. Authors of [44] have proposed solution of QoSaware energy efficient routing solution pertaining to configuration management.

In [45], selection of optimal packet size for improving energy saving in direct communication of WBASNs discussed. In addition, the authors of [46] have addressed the same issue in a communication system developed with WBASN and Wi-Fi network. In [47], authors have considered optimizing total communication energy consumption of WBASN and the Wi-Fi networks using joint data rate adaptation.

Authors of [48] have focused on usage of solar energy harvesting systems for WSN applications [49]. The transmission range changes were chosen as an elementary factor in the many of the existing models [50]. Routing, scheduling of transmissions, synchronizing communication, adapting MAC, and many other such approaches [51] can optimize energy management and lifetime of network, which are key objectives of WSNs. Irrespective of the domain and node mobility of the WSN [51] or the resource cycling models such as solar energy models.

\subsection{Network prototypes}

In [52], authors have proposed ingenious design for assessing the measure respiratory rate, based on acoustic sensor. Microphone places over the neck to record acoustic signals associated with breathing, wherein the band-pass filtered to attain the signal modulations.

Authors of [53], developed in-shoe pressure and acceleration sensors based on classification activities, for postures detection and to understand if the subjects are simultaneously performing armreaching movements.

In [54], authors proposed step-counting mechanism using MEMS, in the case of patients suffering with Parkinson's disease. In [55], authors used wearable sensors for monitoring recovery of patients in post-operative conditions. A varied range of research projects has focused on activity monitoring using wellness applications for increasing exercise compliance. In some of the studies proposed for monitoring lifestyle and clinical intervention issues. In [56], the study has provided an algorithm for detailed analysis of patient activity level, where in the data is recorded as single unit.

Authors of [57] have focused on tri-axial accelerometer oriented custom-designed vest for detecting falls. The emergence of sophisticated smart phones has enabled fall detection systems.

In [58], authors have developed an ear-worn sensor for monitoring the activities and exertion levels in the patients suffering from obstructive pulmonary diseases. Usage of sophisticated machine learning models enabled them to trace varied types of physical activities and improvise them using a worn sensor. In [59], a multi-sensor system evaluating galvanic skin response, skin temperature and heat flow considered for estimating energy consumption. 


\section{Observations and Future Research Direc- tions}

WBASN s are very active and are contemporary domain where there are profound elements and dimensions to be explored for addressing varied levels of architecture and from vivid perspectives of information security. The following set of inputs reflect upon challenges of WBASN s by focusing on distinct set of papers, and the key challenges are depicted in the further sections. Table 1 emphasize the different types of future research problem, which discussed below.

Packet collision in Retransmission: In the instance outburst of stimuli, large volume of network traffic has to flow in a smooth manner ensuring no delay or collisions or any kind of retransmissions. It might be very challenging as routing certain soft components are frugal for WBASN [60].

Utilization of Bandwidth: Profoundly, majority of the novel devices can handle small bytes, and it restricts the movement of data. Hence, there is need for high-level bandwidth for smooth processing from such devices [60].

Time Lag and Storage data collection: The need is to ensure distribution of storage [61].

Synchronization of Time: Complexities might rise in the case of final transmission, challenges in case of time delay and time lag, which might require certain levels of pre and post, processes [62]

Prediction: It is imperative from important data that WBASN should have efficient protocol and algorithm for predicting health problems like heart attack [63] for effective success and health application.

Cost-effectiveness: Though profound developments have taken place in the R\&D of wearable devices, still the cost of such solutions are on high and there is integral need for reducing the price factors [64].

Efficient Prediction Techniques: Existing prediction techniques adapt simple solutions and it could lead to prediction errors. Harvested power is also limited which triggers in thwarting any excessive power spends. Researchers should focus on developing efficient and less error-prone solutions for improving power management [65].

Energy-efficient reliable systems: All the energy-harvesting systems shall envisage benefits from reliable and ultra-reliable energy efficient sensors and nodes. Apart from the anti-fouling coatings over surfaces, reliable sensors thrive to save power and have to be reliable in terms of system longevity. Sensor nodes also increase challenge of calibration and cleansing of the system, which eliminates the need for reducing the quantum of energy required and size of energy system [65].

Storage of Energy: Long lasting and large size applications impact the battery sustenance factors like charge cycles, selfdischarge and environmental conditions. Hence, future researchers have to focus on additional effort for investigating and evaluating performance, reliability of conventional and effective battery solutions [66]. There is potential scope for exploring trade-offs upon usage of batteries and capacitors as the storage solutions [67-75].
Table 1: Table for the Future Research Directions

\begin{tabular}{|c|c|}
\hline $\begin{array}{l}\text { Packet collision in } \\
\text { Retransmission }\end{array}$ & $\begin{array}{l}\text { Packet collision is a big problem in data commu- } \\
\text { nication system. When the router is receiving } \\
\text { data from the multiple nodes there is a chance for } \\
\text { the pocket collisions which pockets are coming } \\
\text { from external nodes, in this condition it is essen- } \\
\text { tial to design the scheduler that eliminates data or } \\
\text { pocket collision [60]. }\end{array}$ \\
\hline $\begin{array}{l}\text { Utilization } \\
\text { Bandwidth }\end{array}$ & $\begin{array}{l}\text { All IoT devices are restricted to limited memory, } \\
\text { so it is challenging to handle the bulk data with } \\
\text { this limited memory [61]. }\end{array}$ \\
\hline $\begin{array}{l}\text { Time Lag and } \\
\text { Storage data col- } \\
\text { lection }\end{array}$ & The need is to ensure distribution of storage [61]. \\
\hline $\begin{array}{l}\text { Synchronization } \\
\text { of Time: }\end{array}$ & $\begin{array}{l}\text { Synchronization of time is a big challenge in the } \\
\text { communication system, when the routers are } \\
\text { receiving the data from one node, it is also man- } \\
\text { datory to receive the data that is coming from } \\
\text { other nodes, so we require developing the syn- } \\
\text { chronization events which collects data without } \\
\text { any losses [62]. }\end{array}$ \\
\hline Prediction & $\begin{array}{l}\text { WBASN should have efficient protocol and } \\
\text { algorithm for predicting health problems like } \\
\text { heart attack [63] for effective success and health } \\
\text { application. }\end{array}$ \\
\hline Cost-effectiveness & $\begin{array}{l}\text { Though profound developments have taken place } \\
\text { in the R\&D of wearable devices, still the cost of } \\
\text { such solutions are on high and there is integral } \\
\text { need for reducing the price factors [64]. }\end{array}$ \\
\hline $\begin{array}{l}\text { Efficient Predic- } \\
\text { tion Techniques: }\end{array}$ & $\begin{array}{l}\text { It is essential to develop the prediction system, } \\
\text { which can identify the battery power problems } \\
\text { and patient's health problems [65]. }\end{array}$ \\
\hline $\begin{array}{l}\text { Energy-efficient } \\
\text { reliable systems }\end{array}$ & $\begin{array}{l}\text { When number sensors are increase in the sensor } \\
\text { node the power consumption of the sensor node } \\
\text { also increases, in this condition it is essential to } \\
\text { develop the power saving application by activat- } \\
\text { ing the sleep mode for the sensors which are not } \\
\text { necessary [65]. }\end{array}$ \\
\hline Storage of Energy & $\begin{array}{l}\text { Future researchers have to focus on additional } \\
\text { effort for investigating and evaluating perfor- } \\
\text { mance, reliability of conventional and effective } \\
\text { battery solutions [66]. }\end{array}$ \\
\hline
\end{tabular}

\section{Conclusion}

WBANs are turning out to be a significant solution and globally there is exponential growth in demand for wearable devices. As such devices are becoming an integral part of monitoring function in varied application systems and domains, the routing protocols of WBASN are gaining significance. Though there are many positives emerging from the process, still certain factors like challenges and complexities of handling are hampering the developments. In development of an effective and quality protocol for routing, right from planning to mitigate impact of external factors over PHY and MAC, to the level of increasing operational efficiency and performance, improving the standards of co-existence, varied factors are to give importance. In this paper, focus is on reviewing taxonomy and literature pertaining to emerging developments in routing protocols and its pros and cons. To derive an outlook on the critical factors essential for handling the WBASN designs. Many of the earlier models and contributions pertaining to QoS reviewed for qualitative comparisons, and it is evident that though there are many models that have evolved in the domain, still there is scope for improvement and operational efficiency in the design and development of effective solutions.

\section{Acknowledgement}

This is a text of acknowledgements. Do not forget people who have assisted you on your work. Do not exaggerate with thanks. If your work has been paid by a Grant, mention the Grant name and number here. 


\section{References}

[1] Ahmadi, Amin, Edmond Mitchell, Francois Destelle, Marc Gowing, Noel E. O'Connor, Chris Richter, and Kieran Moran. "Automatic activity classification and movement assessment during a sports training session using wearable inertial sensors." In Wearable and Implantable Body Sensor Networks (BSN), 2014 11th International Conference on, pp. 98-103. IEEE, 2014.

[2] Penders, Julien, Jef van de Molengraft, Lindsay Brown, Bernard Grundlehner, Bert Gyselinckx, and Chris Van Hoof. "Potential and challenges of body area networks for personal health." In Engineering in Medicine and Biology Society, 2009. EMBC 2009. Annual International Conference of the IEEE, pp. 6569 6572. IEEE, 2009

[3] Malhi, Karandeep, Subhas Chandra Mukhopadhyay, Julia Schnepper, Mathias Haefke, and Hartmut Ewald. "A zigbeebased wearable physiological parameters monitoring system." IEEE sensors journal 12, no. 3 (2012): 423-430.

[4] Poh, Ming-Zher, Kyunghee Kim, Andrew Goessling, Nicholas Swenson, and Rosalind Picard. "Cardiovascular monitoring using earphones and a mobile device." IEEE Pervasive Computing 11, no. 4 (2012): 18-26.

[5] Strohrmann, Christina, Holger Harms, Cornelia Kappeler-Setz, and Gerhard Troster. "Monitoring kinematic changes with fatigue in running using body-worn sensors." IEEE transactions on information technology in biomedicine 16, no. 5 (2012): 983-990.

[6] Hanson, Mark A., Harry C. Powell Jr, Adam T. Barth, Kyle Ringgenberg, Benton H. Calhoun, James H. Aylor, and John Lach. "Body area sensor networks: Challenges and opportunities." Computer 42, no. 1 (2009).

[7] Jeong, Hyocheol, Jeonghyun Kim, and Younghwan Yoo. "Adaptive broadcasting method using neighbor type information in wireless sensor networks." Sensors 11, no. 6 (2011): 5952-5967.

[8] Chen, Min, Sergio Gonzalez, Athanasios Vasilakos, Huasong Cao, and Victor C. Leung. "Body area networks: A survey." Mobile networks and applications 16, no. 2 (2011): 171-193.

[9] Otto, Chris, Aleksandar Milenkovic, Corey Sanders, and Emil Jovanov. "System architecture of a wireless body area sensor network for ubiquitous health monitoring." Journal of mobile multimedia 1, no. 4 (2006): 307-326.

[10] Latré, Benoît, Bart Braem, Ingrid Moerman, Chris Blondia, and Piet Demeester. "A survey on wireless body area networks." Wireless Networks 17, no. 1 (2011): 1-18

[11] Tachtatzis, Christos, Fabio Di Franco, David C. Tracey, Nick F. Timmons, and Jim Morrison. "An energy analysis of IEEE 802.15. 6 scheduled access modes." In GLOBECOM Workshops (GC Wkshps), 2010 IEEE, pp. 1270-1275. IEEE, 2010

[12] Sukor, Marina, Sharifah Ariffin, Norsheila Fisal, SK Syed Yusof, and Adel Abdallah. "Performance study of wireless body area network in medical environment." In Modeling \& Simulation, 2008. AICMS 08. Second Asia International Conference on, pp. 202-206. IEEE, 2008.

[13] Yuce, Mehmet R., and Jamil Khan, eds. Wireless body area networks: Technology, implementation, and applications. CRC Press, 2011.

[14] Galzarano, Stefano, Giancarlo Fortino, and Antonio Liotta. "Embedded self-healing layer for detecting and recovering sensor faults in body sensor networks." In Systems, Man, and Cybernetics (SMC), 2012 IEEE International Conference on, pp. $2377-$ 2382. IEEE, 2012.

[15] Cook, Diane, Kyle D. Feuz, and Narayanan C. Krishnan. "Transfer learning for activity recognition: A survey." Knowledge and information systems 36, no. 3 (2013): 537.

[16] Lo, Benny, and Guang-Zhong Yang. "Body sensor networksresearch challenges and opportunities." (2007): 26-32.

[17] Augimeri, Antonio, Giancarlo Fortino, Stefano Galzarano, and Raffaele Gravina. "Collaborative body sensor networks." In Systems, Man, and Cybernetics (SMC), 2011 IEEE International Conference on, pp. 3427-3432. IEEE, 2011.

[18] Fortino, Giancarlo, Stefano Galzarano, Raffaele Gravina, and Wenfeng Li. "A framework for collaborative computing and multi-sensor data fusion in body sensor networks." Information Fusion 22 (2015): 50-70.

[19] Fortino, Giancarlo, Roberta Giannantonio, Raffaele Gravina, Philip Kuryloski, and Roozbeh Jafari. "Enabling effective programming and flexible management of efficient body sensor network applications." IEEE Transactions on Human-Machine Systems 43, no. 1 (2013): 115-133.
[20] Augimeri, Antonio, Giancarlo Fortino, Manoj R. Rege, Vlado Handziski, and Adam Wolisz. "A cooperative approach for handshake detection based on body sensor networks." In Systems Man and Cybernetics (SMC), 2010 IEEE International Conference on, pp. 281-288. IEEE, 2010.

[21] Fortino, Giancarlo, Daniele Parisi, Vincenzo Pirrone, and Giuseppe Di Fatta. "BodyCloud: A SaaS approach for community body sensor networks." Future Generation Computer Systems 35 (2014): 62-79.

[22] Huang, Yueh-Min, Meng-Yen Hsieh, Han-Chieh Chao, Shu-Hui Hung, and Jong Hyuk Park. "Pervasive, secure access to a hierarchical sensor-based healthcare monitoring architecture in wireless heterogeneous networks." IEEE journal on selected areas in communications 27, no. 4 (2009).

[23] Wang, Changhong, Qiang Wang, and Shunzhong Shi. "A distributed wireless body area network for medical supervision." In Instrumentation and Measurement Technology Conference (I2MTC), 2012 IEEE International, pp. 2612-2616. IEEE, 2012.

[24] Wolf, Lars, and Sana Saadaoui. "Architecture concept of a wireless body area sensor network for health monitoring of elderly people." In Consumer Communications and Networking Conference, 2007. CCNC 2007. 4th IEEE, pp. 722-726. IEEE, 2007.

[25] Mukhopadhyay, Subhas Chandra. "Wearable sensors for human activity monitoring: A review." IEEE sensors journal 15, no. 3 (2015): 1321-1330.

[26] Bag, Anirban, and Mostafa A. Bassiouni. "Energy efficient thermal aware routing algorithms for embedded biomedical sensor networks." In Mobile Adhoc and Sensor Systems (MASS), 2006 IEEE International Conference on, pp. 604-609. IEEE, 2006.

[27] Watteyne, Thomas, Isabelle Augé-Blum, Mischa Dohler, and Dominique Barthel. "Anybody: a self-organization protocol for body area networks." In Proceedings of the ICST 2nd international conference on Body area networks, p. 6. ICST (Institute for Computer Sciences, Social-Informatics and Telecommunications Engineering), 2007.

[28] Moh, Melody, Benjamin Jack Culpepper, Lan Dung, TengSheng Moh, Takeo Hamada, and Ching-Fong Su. "On data gathering protocols for in-body biomedical sensor networks." In Global Telecommunications Conference, 2005. GLOBECOM'05. IEEE, vol. 5, pp. 6-pp. IEEE, 2005.

[29] Movassaghi, Samaneh, Mehran Abolhasan, and Justin Lipman. "Energy efficient thermal and power aware (ETPA) routing in body area networks." In Personal Indoor and Mobile Radio Communications (PIMRC), 2012 IEEE 23rd International Symposium on, pp. 1108-1113. IEEE, 2012.

[30] Braem, Bart, Benoit Latre, Ingrid Moerman, Chris Blondia, and Piet Demeester. "The wireless autonomous spanning tree protocol for multihop wireless body area networks." In Mobile and Ubiquitous Systems: Networking \& Services, 2006 Third Annual International Conference on, pp. 1-8. IEEE, 2006.

[31] Braem, Bart, Benoît Latré, Chris Blondia, Ingrid Moerman, and Piet Demeester. "Improving reliability in multi-hop body sensor networks." In Sensor Technologies and Applications, 2008. SENSORCOMM'08. Second International Conference on, pp. 342-347. IEEE, 2008.

[32] Ruzzelli, Antonio G., Raja Jurdak, Gregory MP O'Hare, and Peter Van Der Stok. "Energy-efficient multi-hop medical sensor networking." In Proceedings of the 1st ACM SIGMOBILE international workshop on Systems and networking support for healthcare and assisted living environments, pp. 37-42. ACM, 2007

[33] Bag, Anirban, and Mostafa A. Bassiouni. "Biocomm-A crosslayer medium access control (MAC) and routing protocol codesign for biomedical sensor networks." International Journal of Parallel, Emergent and Distributed Systems 24, no. 1 (2009): 85103.

[34] Djenouri, Djamel, and Ilangko Balasingham. "New QoS and geographical routing in wireless biomedical sensor networks." In Broadband Communications, Networks, and Systems, 2009. BROADNETS 2009. Sixth International Conference on, pp. 1-8. IEEE, 2009.

[35] Penders, Julien, Valer Pop, L. Caballero, Jef van de Molengraft, Rob van Schaijk, Ruud Vullers, and Chris Van Hoof. "Power optimization in body sensor networks: The case of an autonomous wireless EMG sensor powered by PV-cells." In Engineering in Medicine and Biology Society (EMBC), 2010 Annual International Conference of the IEEE, pp. 2017-2020. IEEE, 2010.

[36] Bae, Joonsung, Long Yan, and Hoi-Jun Yoo. "A low energy injection-locked FSK transceiver with frequency-to-amplitude 
conversion for body sensor applications." IEEE Journal of Solidstate circuits 46, no. 4 (2011): 928-937.

[37] Yoo, Hoi-Jun, Namjun Cho, and Jerald Yoo. "Low energy wearable body-sensor-network." In Engineering in Medicine and $\mathrm{Bi}$ ology Society, 2009. EMBC 2009. Annual International Conference of the IEEE, pp. 3209-3212. IEEE, 2009.

[38] Yoo, Hoi-Jun, Seong-Jun Song, Namjun Cho, and Hye-Jeong Kim. "Low energy on-body communication for BSN." In 4th International Workshop on Wearable and Implantable Body Sensor Networks (BSN 2007), pp. 15-20. Springer Berlin Heidelberg, 2007.

[39] Bui, Francis Minhthang, and Dimitrios Hatzinakos. "Quality of service regulation in secure body area networks: System modeling and adaptation methods." EURASIP Journal on Wireless Communications and Networking 2011 (2011): 3.

[40] Elias, Jocelyne. "Optimal design of energy-efficient and costeffective wireless body area networks." Ad Hoc Networks 13 (2014): 560-574.

[41] Razzaque, Md Abdur, Choong Seon Hong, and Sungwon Lee. "Data-centric multi-objective QoS-aware routing protocol for body sensor networks." Sensors 11, no. 1 (2011): 917-937.

[42] Latre, Benoit, Bart Braem, Ingrid Moerman, Chris Blondia, Elisabeth Reusens, Wout Joseph, and Piet Demeester. "A low-delay protocol for Multihop wireless body area networks." In Mobile and Ubiquitous Systems: Networking \& Services, 2007. Mobi Quitous 2007. Fourth Annual International Conference on, pp. 18. IEEE, 2007.

[43] Liang, Xuedong, Ilangko Balasingham, and Sang-Seon Byun. "A reinforcement learning based routing protocol with QoS support for biomedical sensor networks." In Applied Sciences on Biomedical and Communication Technologies, 2008. ISABEL'08. First International Symposium on, pp. 1-5. IEEE, 2008.

[44] Seo, Su-Ho, Sai Anand Gopalan, Seung-Man Chun, Ki-Jung Seok, Jae-Wook Nah, and Jong-Tae Park. "An energy-efficient configuration management for multi-hop wireless body area networks." In Broadband network and multimedia technology (IC-BNMT), 2010 3rd IEEE international conference on, pp. 1235-1239. IEEE, 2010

[45] Domingo, Mari Carmen. "Packet size optimization for improving the energy efficiency in body sensor networks." ETRI Journal 33, no. 3 (2011): 299-309.

[46] Li, Yantao, Xin Qi, Zhen Ren, Gang Zhou, Di Xiao, and Shaojiang Deng. "Energy modeling and optimization through joint packet size analysis of BSN and WiFi networks." In Performance Computing and Communications Conference (IPCCC), 2011 IEEE 30th International, pp. 1-8. IEEE, 2011.

[47] Li, Yantao, Ge Peng, Xin Qi, Gang Zhou, Di Xiao, Shaojiang Deng, and Hongyu Huang. "Towards energy optimization using joint data rate adaptation for BSN and WiFi networks." In Networking, Architecture and Storage (NAS), 2012 IEEE 7th International Conference on, pp. 235-244. IEEE, 2012.

[48] Yaakob, Naimah, and Ibrahim Khalil. "Packet size optimization for congestion control in pervasive healthcare monitoring." In Information Technology and Applications in Biomedicine (ITAB), 2010 10th IEEE International Conference on, pp. 1-4. IEEE, 2010.

[49] Buchli, Bernhard, Felix Sutton, Jan Beutel, and Lothar Thiele. "Towards enabling uninterrupted long-term operation of solar energy harvesting embedded systems." In European Conference on Wireless Sensor Networks, pp. 66-83. Springer, Cham, 2014.

[50] Noh, Dong Kun. "Transmission range determination with a timeslot-based energy distribution scheme for solar-energy harvesting sensor systems." In Future Information Communication Technology and Applications, pp. 661-669. Springer Netherlands, 2013.

[51] El Korbi, Inès, and Sherali Zeadally. "Energy-aware sensor node relocation in mobile sensor networks." Ad Hoc Networks 16 (2014): 247-265.

[52] Corbishley, Phil, and Esther Rodríguez-Villegas. "Breathing detection: towards a miniaturized, wearable, battery-operated monitoring system." IEEE Transactions on Biomedical Engineering 55, no. 1 (2008): 196-204

[53] Sazonov, Edward S., George Fulk, Nadezhda Sazonova, and Stephanie Schuckers. "Automatic recognition of postures and activities in stroke patients." In Engineering in Medicine and Biology Society, 2009. EMBC 2009. Annual International Conference of the IEEE, pp. 2200-2203. IEEE, 2009.

[54] Giansanti, Daniele, Giovanni Maccioni, and Sandra Morelli. "An experience of health technology assessment in new models of care for subjects with Parkinson's disease by means of a new wearable device." TELEMEDICINE and e-HEALTH 14, no. 5 (2008): 467-472.

[55] Aziz, Omer, L. Atallah, B. Lo, M. ElHelw, L. Wang, GuangZhong Yang, and A. Darzi. "A pervasive body sensor network for measuring postoperative recovery at home." Surgical innovation 14, no. 2 (2007): 83-90.

[56] Hecht, Ariel, Shuyi Ma, Janos Porszasz, Richard Casaburi, and COPD Clinical Research Network. "Methodology for using long-term accelerometer monitoring to describe daily activity patterns in COPD." COPD: Journal of Chronic Obstructive Pulmonary Disease 6, no. 2 (2009): 121-129.

[57] Bourke, Alan K., Pepijn WJ Van de Ven, Amy E. Chaya, Gearóid M. OLaighin, and John Nelson. "Testing of a long-term fall detection system incorporated into a custom vest for the elderly." In Engineering in Medicine and Biology Society, 2008. EMBS 2008. 30th Annual International Conference of the IEEE, pp. 2844-2847. IEEE, 2008.

[58] Patel, Shyamal, Hyung Park, Paolo Bonato, Leighton Chan, and Mary Rodgers. "A review of wearable sensors and systems with application in rehabilitation." Journal of neuro engineering and rehabilitation 9, no. 1 (2012): 21.

[59] Patel, Sanjay A., Roberto P. Benzo, William A. Slivka, and Frank C. Sciurba. "Activity monitoring and energy expenditure in COPD patients: a validation study." COPD: Journal of Chronic Obstructive Pulmonary Disease 4, no. 2 (2007): 107-112.

[60] Xia, Lingli, Stephen Redfield, and Patrick Chiang. "Experimental characterization of a UWB channel for body area networks." EURASIP Journal on Wireless Communications and Networking 2011 (2011): 4.

[61] Le, Trung Q., Changqing Cheng, Akkarapol Sangasoongsong, Woranat Wongdhamma, and Satish TS Bukkapatnam. "Wireless wearable multisensory suite and real-time prediction of obstructive sleep apnea episodes." IEEE journal of translational engineering in health and medicine 1 (2013): 2700109-2700109.

[62] Quwaider, Muhannad, Mahmoud Taghizadeh, and Subir Biswas. "Modeling on-body dtn packet routing delay in the presence of postural disconnections." EURASIP journal on wireless communications and networking 2011, no. 1 (2010): 280324.

[63] Li, Huaming, and Jindong Tan. "Heartbeat-driven mediumaccess control for body sensor networks." IEEE Transactions on Information Technology in Biomedicine 14, no. 1 (2010): 44-51.

[64] Bring Down Prices of Wearable Devices. [Online]. Available: http://www.eetindia.co.in/ART_8800703467_1800007_NT_c8cc 7af5. HTM, accessed Sep. 28, 2014.

[65] Shaikh, Faisal Karim, and Sherali Zeadally. "Energy harvesting in wireless sensor networks: A comprehensive review." Renewable and Sustainable Energy Reviews 55 (2016): 1041-1054.

[66] Zeadally, Sherali, Samee Ullah Khan, and Naveen Chilamkurti. "Energy-efficient networking: past, present, and future." The Journal of Supercomputing (2012): 1-26.

[67] RAMAN, Y.S.V., MADHAV, B.T.P., MOUNIKA, G., SAI TEJA, K., SAI KUMAR, S.B.V.N. and SRI HARSHA, K., 2016. Analysis of circularly polarized notch band antenna with DGS. ARPN Journal of Engineering and Applied Sciences, 11(17), pp. 10140-10150.

[68] MADHAV, B.T.P., CHHATKULI, S., MANIKANTAPRASANTH, A., BHARGAV, Y., DINESH NAGA VENKATA SAI, U. and FEERAZ, S., 2014. Measurement of dimensional characteristics of microstrip antenna based on mathematical formulation. International Journal of Applied Engineering Research, 9(9), pp. 1063-1074.

[69] MADHAV, B.T.P., MOHAN REDDY, S.S., SHARMA, N., RAVINDRANATH CHOWDARY, J., PAVITHRA, B.R., KISHORE, K.N.V.S., SRIRAM, G. and SACHIN KUMAR, B., 2013. Performance characterization of radial stub microstrip bow-tie antenna. International Journal of Engineering and Technology, 5(2), pp. 760-764.

[70] SRINIVAS, M.S.S.S., RAMAKRISHNA, T.V., MADHAV, B.T.P., BHAGYALAKSHMI, N., MADHAVI, S. and VENKATESWARULU, K., 2015. A novel compact CPW fed slot antenna with EBG structure. ARPN Journal of Engineering and Applied Sciences, 10(2), pp. 835-841.

[71] RAMKIRAN, D.S., MADHAV, B.T.P., PRASANTH, A.M., HARSHA, N.S., VARDHAN, V., AVINASH, K., CHAITANYA, M.N. and NAGASAI, U.S., 2015. Novel compact asymmetrical fractal aperture Notch band antenna. Leonardo Electronic Journal of Practices and Technologies, 14(27), pp. 112.

[72] SUNDER, P.S., KOTAMRAJU, S.K., RAMAKRISHNA, T.V., MADHAV, B.T.P., SRUTHI, T.S., VIVEK, P., KUMAR, J.J. 
and DILEEP, M., 2015. Novel miniatured wide band annular slot monopole antenna. Far East Journal of Electronics and Communications, 14(2), pp. 149-159.

[73] AJAY BABU, M., MADHAV, B.T.P., NAGA VAISHNAVI, D., RADHAKRISHNA, P., BHARATH, N., MADHURI, K. BHAVANI PRASAD, K. and HARISH, K., 2015. Flared Vshape slotted monopole multiband antenna with metamateria loading. International Journal on Communications Antenna and Propagation, 5(2), pp. 93-97.

[74] MADHAV, B.T.P., KAZA, H., VAKA, J.K., SRAVAN KUMAR, K., SRIHARSHA, N., JASWANTH KUMAR, J., SIDDHARTH, D.S. and SAI TEJA REDDY, D., 2015. Design and analysis of compact coplanar wave guide fed asymmetric monopole antennas. Research Journal of Applied Sciences, Engineering and Technology, 10(3), pp. 247-252.

[75] MADHAV, B.T.P., KHAN, H. and KOTAMRAJU, S.K., 2016. Circularly polarized slotted aperture antenna with coplanar waveguide fed for broadband applications. Journal of Engineering Science and Technology, 11(2), pp. 267-277. 